\title{
Uso de sellantes de fosas y fisuras para la prevención de caries en población infanto-juvenil: Revisión metodológica de ensayos clínicos
}

\author{
Use of pit and fissure sealants for preventing caries in child \\ population: Methodological review of clinical trials
}

\author{
Faleiros Chioca $S^{1}$, Urzúa Araya I ${ }^{2}$, Rodríguez Martínez $\mathrm{G}^{2}$, Cabello Ibacache $\mathrm{R}^{2}$
}

\begin{abstract}
RESUMEN
La caries dental es uno de los problemas más relevantes dentro del ámbito odontológico y de la Salud Pública. En Chile existe una alta prevalencia de caries en la población infanto-juvenil, siendo esta una de las prioridades de salud del país. Se constata la necesidad de utilizar métodos complementarios para su control. Los sellantes de fosas y fisuras representan una de las intervenciones preventivas más efectivas en odontología, siendo considerados como un aporte a la Salud Pública. Sin embargo, no existen evidencias consistentes acerca de su verdadera efectividad e impacto en la prevención de caries. Las inconsistencias encontradas podrían deberse a la presencia de sesgos o a la heterogeneidad de los estudios disponibles en la literatura. Así, se propone realizar una revisión sistemática con el fin de evaluar la calidad metodológica de los ensayos clínicos controlados que utilizan sellantes de fosas y fisuras. Se condujo una búsqueda de ensayos realizados entre 1990 y 2007, encontrándose trece que cumplieron con los criterios de inclusión y exclusión. Los estudios incluidos fueron obtenidos y evaluados por un único revisor. Se concluye que la evidencia que avala la utilización de esta intervención es metodológicamente muy pobre. Estos hallazgos debieran estimular a los profesionales a esclarecer la verdad referente a la efectividad de los sellantes, antes de aplicarlos indiscriminadamente. Se recomienda la realización de nuevos ensayos clínicos ejecutados con un adecuado diseño metodológico y de análisis estadístico, cuyos resultados sirvan como evidencia confiable para la toma de decisiones que guíen las políticas de Salud Pública.
\end{abstract}

Rev. Clin. Periodoncia Implantol. Rehabil. Oral Vol. 6(1); 14-19, 2013.

Palabras clave: Epidemiología, caries dental, sellantes de fosas y fisuras.

\begin{abstract}
Dental decay is one of the most important problems within the Public Oral Health. Chile has a high prevalence of decay among children and adolescents, being this issue one of the priorities of public health in the country. Taking into account this context, there is a necessity of using complementary methods for its control. Pit and fissures sealants represent one of the most effective preventive interventions in dentistry, being considered as a contribution to the public health interventions. Nevertheless, there are no consistent evidences about their true effectiveness and impact in the prevention of caries. These inconsistencies could be due to the presence of bias or the heterogeneity of the studies available in literature. Thus, we propose to make a review with the aim to evaluate the methodological quality of clinical trials using pit and fissure sealants. A search of studies between 1990 and 2012 was conducted and 18 clinical trials that fulfilled the inclusion and exclusion criteria were found. The studies included were obtained and evaluated by a single reviewer. This review concludes that the evidence that guarantees the use of this intervention is methodologically poor. These findings may stimulate the professionals to clarify the truth referring to the effectiveness of sealants, before applying it indiscriminately. The accomplishment of new clinical trials executed with an appropriate methodological design and statistical analysis is recommended, generating reliable results for the decision making that guides the Public Health Policies.
\end{abstract}

Rev. Clin. Periodoncia Implantol. Rehabil. Oral Vol. 6(1); 14-19, 2013.

Key words: Epidemiology, dental caries, pit and fissure sealants.

\section{INTRODUCCIÓN}

La caries dental es uno de los problemas más relevantes dentro del ámbito odontológico y de la Salud Pública. Según la Organización Mundial de la Salud, entre el 60 a $90 \%$ de los niños del mundo presentan caries. Por otro lado, los cuidados dentales curativos en los países industrializados representan una significativa carga económica, donde el 5 a $10 \%$ de los gastos en Salud Pública se relacionan con la salud oral. Los cuidados curativos son más costosos en comparación con las intervenciones preventivas ${ }^{(1,2)}$. En la mayoría de los países en vías de desarrollo más del $90 \%$ de las caries se encuentran sin tratamiento ${ }^{(3)}$.
La importante prevalencia de caries ha sido también detectada en Chile, por medio de estudios nacionales, realizados en los años 19961999 y $2007^{(4,5)}$. Aun así, el último estudio epidemiológico nacional (2007) mostró la persistencia de una alta proporción de caries en la población infantil, con una prevalencia nacional en niños(as) de 6 años de $70.3 \%{ }^{(5)}$. Pese a que existen muchos programas de prevención tales como la fluoruración de aguas y educación en salud bucal, entre otras actividades, se constata la necesidad de utilizar métodos complementarios para el control de la enfermedad. Actualmente, el Ministerio de Salud considera la prevención de lesiones de caries como una de las prioridades de salud del país, focalizando sus actividades en la población menor de 20 años.

1. Doctorado en Salud Pública. Departamento de Odontología Restauradora. Facultad de Odontología, Universidad de Chile. Chile.

2. Magíster en Ciencias Odontológicas. Departamento de Odontología Restauradora. Facultad de Odontología, Universidad de Chile. Chile. 
Se cree que los sellantes de fosas y fisuras, ampliamente utilizados en programas comunitarios de control de caries, son uno de los materiales más efectivos para este fin ${ }^{(6)}$. Su importancia se debe a que las superficies oclusales de los molares son especialmente susceptibles a la acumulación de placa bacteriana y al consecuente desarrollo de caries, debido a su irregular anatomía. Los sellantes actúan eliminando esta irregularidad, lo que dificulta la adhesión bacteriana y facilita la higiene ${ }^{(7,8)}$.

Existen dos tipos disponibles de sellantes: En base a resina y en base a vidrio ionómero, distinguiéndose entre sí por su mecanismo de polimerización y adhesión a la estructura dental ${ }^{(8)}$. Además, el segundo material presenta la supuesta ventaja de funcionar como reservorio de flúor, ya que presenta fluoruro en su composición química ${ }^{(9)}$. Por tratarse de una intervención de aplicación fácil, atraumática, indolora y bien tolerada, se considera como un aporte relevante a la Salud Pública, especialmente en la población infantil. En Chile, la utilización de sellantes de puntos y fisuras es una estrategia que está sugerida en los documentos de orientación clínica y guías basadas en evidencia que se encuentran disponibles para dar atención odontológica a la población infanto juvenil. Es por tanto de interés conocer los resultados de la literatura científica en este sentido para este grupo de edad en particular.

Ahovuo-Saloranta y col.(10), en el año 2008 publican una revisión sistemática de la literatura basados en la metodología de la Librería Cochrane. En ella intentan comparar el rendimiento en la prevención de desarrollo de nuevas lesiones de caries entre superficies selladas y no selladas, además de establecer diferencias entre los distintos materiales empleados para este fin. El autor concluye que la efectividad de los sellantes de fosas y fisuras es obvia en poblaciones de alto riesgo, mientras que en otras condiciones de riesgo es deficiente. Por otro lado, los resultados de esta revisión en cuanto a los distintos materiales utilizados como sellantes de fosas y fisuras, son conflictivos. En este sentido, se han reportado resultados inconsistentes con respecto al uso de los sellantes como método preventivo de caries en superficies oclusales utilizando distintos materiales. Algunos estudios que compararon la efectividad de sellantes de resina con sellantes de vidrio ionómero no detectaron diferencias en la incidencia de caries o un mejor efecto protector para el grupo del vidrio ionómero, aún tomando en cuenta que la retención de este material es inferior al de sellante con base a resina ${ }^{(11)}$. Por otro lado, se han encontrado estudios donde se reportan resultados superiores respecto a la prevención de caries para los sellantes de resina ${ }^{(12,13)}$. Las inconsistencias encontradas podrían deberse a la presencia de sesgos o a una posible heterogeneidad metodológica de los estudios.

Una de las formas directas para evaluar la calidad metodológica en el área de las ciencias odontológicas, consiste en revisar la producción científica para la prevención y tratamiento de sus patologías más importantes. De esta manera, el objetivo de la presente revisión consiste en evaluar la calidad metodológica de los ensayos clínicos controlados que utilizan sellantes de fosas y fisuras para la prevención de caries dental.

\section{MATERIALES Y MÉTODOS}

\section{Criterios para la Selección de Estudios}

Se realizó una revisión metodológica de ensayos clínicos controlados que compararon la efectividad entre los distintos tipos de sellantes. Se incluyeron estudios con diseño de boca dividida o con dos o más grupos de comparación, publicados en idioma inglés, español o portugués. Los ensayos incluidos comprendieron solo a la población menor de 18 años al comienzo del estudio y publicados entre enero del año 1990 hasta 2012. La justificación de la inclusión de los estudios desde el año 1990 se debe a que en las dos últimas décadas se experimentaron importantes instancias de desarrollo tecnológico de los materiales y técnicas de aplicación. Adicionalmente, se consideraron solamente estudios donde las piezas dentales estuvieran libres de caries cavitadas al comienzo del tiempo de seguimiento.

Quedaron excluidos de esta revisión los ensayos cuyo desenlace primario fue la sobrevida del material, así como los estudios cuasi-experimentales, estudios de cohorte, relatos de casos clínicos, ensayos realizados in vitro y estudios de seguimiento posterior a la ejecución de un ensayo clínico original.

\section{Estrategia de Búsqueda}

Se condujo una búsqueda en las bases de datos MEDLINE y LILACS, en diferentes textos de odontología preventiva y restauradora, además de tesis relacionadas.

Los principales términos de búsqueda para la presente revisión fueron una combinación de: Caries sealant and clinical trial/randomized clinical trial/controled clinical trial/caries sealant and comparative study/ selantes de fóssulas e fissuras and ensaio clínico controlado or estudo comparativo/sellantes de fosas y fisuras (selladores) and ensayo clínico controlado/ensayo clínico controlado randomizado (aleatorizado) or estudio comparativo.

El término "estudio comparativo", se utilizó en la búsqueda por ser ampliamente utilizado en el área de investigación odontológica.

\section{Extracción de la Información y Calidad Metodológica}

Los estudios incluidos fueron obtenidos y evaluados por un único revisor, mediante el uso de un formulario para la evaluación crítica de la calidad metodológica recomendado por el grupo CONSORT ${ }^{(13)}$ y otros textos relacionados ${ }^{(14,15)}$. Los principales puntos evaluados en cada estudio se presentan en las Tablas 1 y 2 .

\section{Análisis de los Datos Obtenidos}

Los resultados de los estudios seleccionados y analizados se presentan narrativamente en tablas de resumen (Tablas 3 y 4 ).

\section{RESULTADOS}

Se encontraron 18 ensayos clínicos que evaluaron la efectividad en el control de caries de los sellantes de fosas y fisuras (Figura 1). En 12 de ellos se comparó el sellante de resina con el sellante de vidrio ionómero convencional, en 2 de ellos se comparó el sellante de vidrio ionómero convencional con el de vidrio ionómero modificado por resina, en 3 de ellos se compararon distintas marcas de sellantes de resina, y en 1 de ellos se comparó un sellante de resina con un grupo control.

En la Tabla 3 se muestra la evaluación metodológica de los estudios contestando las preguntas planteadas en la Tabla 1, asignándole un símbolo positivo $(+)$ si se responde adecuadamente a la pregunta planteada o con un símbolo negativo (-) en caso contrario.

En la Tabla 4 se presenta la evaluación de reporte de resultados de los estudios según las preguntas planteadas por la Tabla 2 , siguiendo el mismo procedimiento aplicado anteriormente. Además, en las tablas de resultados se presenta el porcentaje de incumplimiento de las preguntas planteadas en cada estudio y, a su vez, el porcentaje de incumplimiento de cada aspecto evaluado individualmente.

\section{Tabla 1. Puntos para la evaluación metodológica.}

\begin{tabular}{ll}
\hline $\mathrm{H}^{*}$ & ¿Estuvo claramente definida la hipótesis u objetivo del estudio? \\
$\mathrm{CB}$ & ¿E stuvieron claramente definidas las caracteristicas basales de la población? \\
$\mathrm{CI}$ & ¿Se expresaron adecuadamente los criterios de inclusión y exclusión? \\
$\mathrm{TM}$ & ¿Se procedió a la estimación del tamaño muestral? \\
$\mathrm{TS}$ & ¿Hubo un adecuado tiempo de seguimiento para pesquisar el desenlace? \\
$\mathrm{PS}$ & ¿Se describieron las pér didas de seguimiento? \\
$\mathrm{PP}$ & ¿El porcentaje de pérdidas fue menor al $10 \% ?$ \\
$\mathrm{DI}$ & ¿Hubo una descripción detallada de las intervenciones? \\
$\mathrm{CD}$ & ¿Estuvieron claramente expresados los criterios diagnósticos para el desenlace? \\
$\mathrm{CA}$ & ¿Hubo capacitación previa de los clínicos participantes? \\
$\mathrm{CO}$ & ¿Se pesquisó la presencia de co-intervención? \\
EN & ¿Se utilizó algún procedimiento de enmascaramiento? \\
A & ¿Los participantes fueron asignados aleatoriamente? \\
-Abreviatura de la pregunta planteada.
\end{tabular}

Tabla 2. Puntos para la evaluación de análisis de datos y reporte de resultados.

$M A^{*}$ ¿Se expresan los resultados a través de alguna medida de asociación o de impacto como RR, OR, NNT oRAR?

AIT ¿Hubo un análisis por intención de tratamiento?

IC ¿Se expresaron los resultados con su respectivo intervalo de confianza?

P ¿Se expresó el valor p de significación estadistica?

AM ¿Se realizó análisis multinivel? (si el contexto lo ameritaba)

Col ¿Se consideró la presencia de co-intervención en el análisis?

- Abreviatura de la pregunta planteada. RR =Riesgo Relativo; OR =Odds Ratio (Razón de Productos Cruzados); NNT = Numero Necesario de Pacientes a Tratar; RAR = Reducción Absoluta de Riesgo. 
Tabla 3. Evaluación metodológica de los estudios incluidos según criterios de la Tabla 1.

\begin{tabular}{|c|c|c|c|c|c|c|c|c|c|c|c|c|c|c|c|c|c|}
\hline Autor & Referencia & $\begin{array}{l}\text { Edad } \\
\text { (años) }\end{array}$ & Intervenciones & TM & $\mathrm{CB}$ & $\mathrm{Cl}$ & TS & PS & PP & DI & $C D$ & CA & CO & EN & A & Res & $\mathrm{Pl} \%$ \\
\hline Beiruti & 17 & 7 & $\mathrm{R} / \mathrm{VI}$ & 103 & - & + & + & + & - & + & + & + & - & - & + & $\mathrm{VI}$ & 33.3 \\
\hline Poulsen S. & 18 & $5-16$ & $\mathrm{R} / \mathrm{Vl}$ & 153 & - & + & + & - & - & + & + & + & - & - & - & $\mathrm{R}$ & 50.0 \\
\hline Pardi V. & 18 & 6 & $\mathrm{VI} / \mathrm{VIm} / \mathrm{C}$ & 208 & - & + & + & + & - & + & + & + & - & - & + & $\mathrm{VI}$ & 33.3 \\
\hline Poulsen S. & 13 & 7 & $\mathrm{R} / \mathrm{VI}$ & 170 & - & + & + & + & - & + & + & + & - & - & + & $\mathrm{R}$ & 33.3 \\
\hline Villela L. & 20 & $10-14$ & VI/VIm & 12 & - & + & + & + & + & + & - & - & - & - & + & NS & 41.6 \\
\hline Mark M. & 21 & $7-10$ & $\mathrm{R} / \mathrm{VI}$ & 50 & - & + & + & + & - & + & - & - & + & - & - & NS & 50.0 \\
\hline Silva R. & 22 & $?^{2}$ & $\mathrm{Ra} / \mathrm{Rf}$ & 27 & - & + & + & + & - & + & - & + & - & + & - & NS & 41.6 \\
\hline Arrow $\mathrm{P}$. & 23 & 7 & $\mathrm{R} / \mathrm{VI}$ & 465 & - & + & + & + & - & + & + & + & - & - & + & $\mathrm{Vl}$ & 33.3 \\
\hline Soung Y. & 24 & $7-8$ & $\mathrm{R} / \mathrm{VI}$ & 2018 & + & + & + & + & - & + & + & + & - & - & + & $\mathrm{R}$ & 25.0 \\
\hline Zuanon A. & 25 & $7-11$ & $5 R / C^{*}$ & 29 & - & + & - & - & - & + & + & - & - & - & - & NS & 66.6 \\
\hline Karlzen G. & 11 & 7 & $\mathrm{R} / \mathrm{VI}$ & 47 & - & + & - & + & + & + & - & - & - & - & + & $\mathrm{VI}$ & 50.0 \\
\hline Nóbrega P. & 26 & $6-9$ & $3 R / C^{* *}$ & 74 & - & + & - & + & + & + & + & - & - & - & + & $\mathrm{R}^{\star \star *}$ & 50.0 \\
\hline Forrs $\mathrm{H}$. & 27 & $6-14$ & $\mathrm{R} / \mathrm{VI}$ & 166 & - & + & + & + & + & + & + & + & - & - & + & NS & 25.0 \\
\hline Barja F. & 28 & $5-8$ & $\mathrm{R} / \mathrm{VI}$ & 36 & - & + & + & - & - & + & + & + & - & + & + & NS & 41.6 \\
\hline Kervanto S. & 29 & $12-16$ & $\mathrm{R} / \mathrm{VI}$ & 559 & - & + & + & - & - & + & + & + & - & - & + & $\mathrm{R}$ & 50.0 \\
\hline Dhar V. & 30 & $6-10$ & $\mathrm{R} / \mathrm{VI}$ & 25 & - & + & + & + & + & + & - & + & - & - & - & $\mathrm{VI}$ & 50.0 \\
\hline Antonson S. & 31 & $5-9$ & $\mathrm{R} / \mathrm{VI}$ & 39 & + & + & + & + & + & + & - & + & - & + & - & NS & 33.3 \\
\hline Borges B. & 32 & $12-19$ & $\mathrm{R} / \mathrm{C}$ & 29 & + & + & + & - & - & + & + & + & - & - & + & $\mathrm{R}$ & 41.6 \\
\hline & & & $\mathrm{Pl} \%$ & - & 83.3 & 0 & 16.6 & 27.7 & 66.6 & 0 & 33.3 & 27.7 & 94.4 & 83.3 & 33.3 & - & \\
\hline
\end{tabular}

Tabla 4. Evaluación de análisis de datos y reporte de resultados de los estudios incluidos según criterios de la Tabla 2.

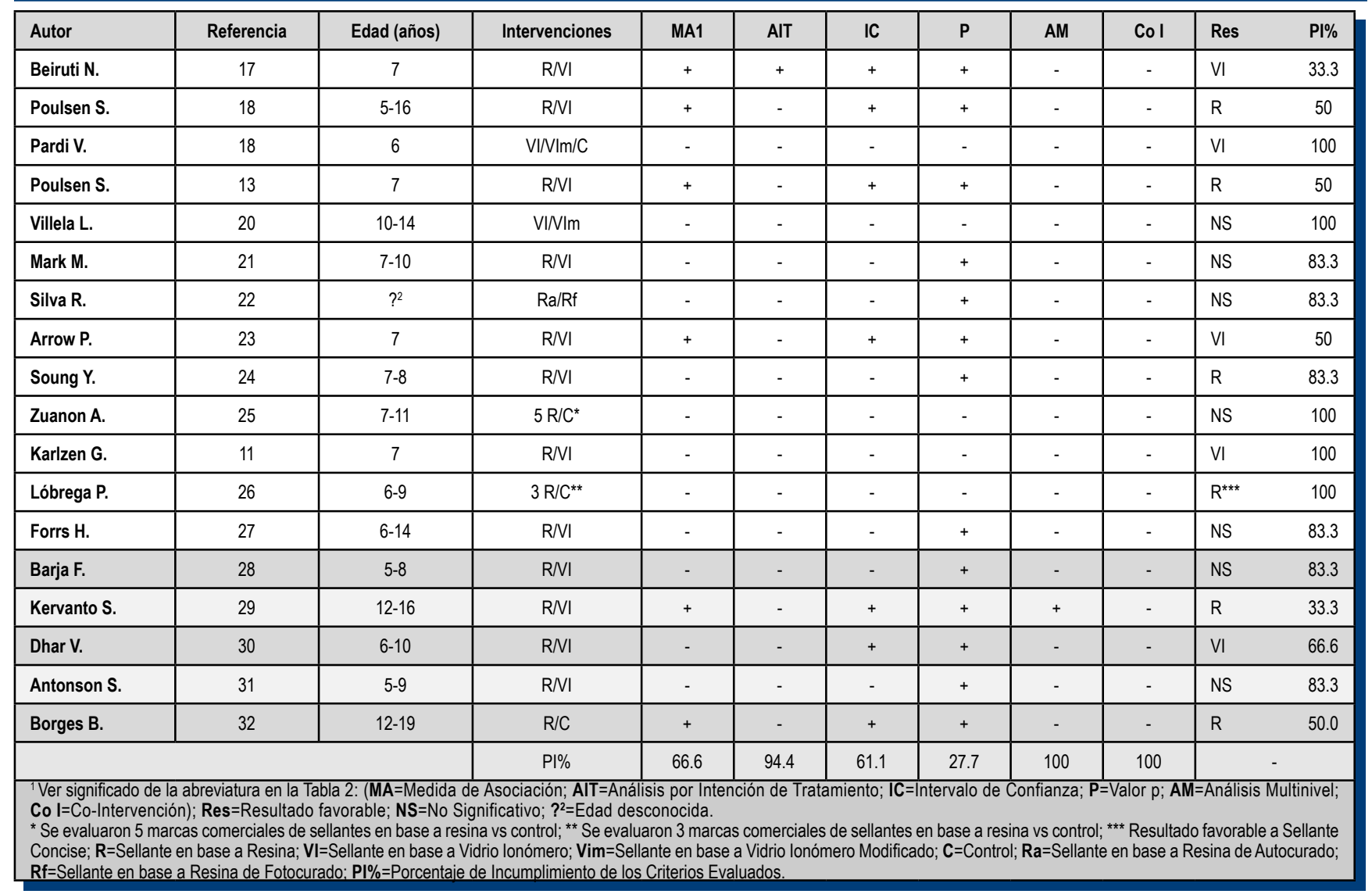




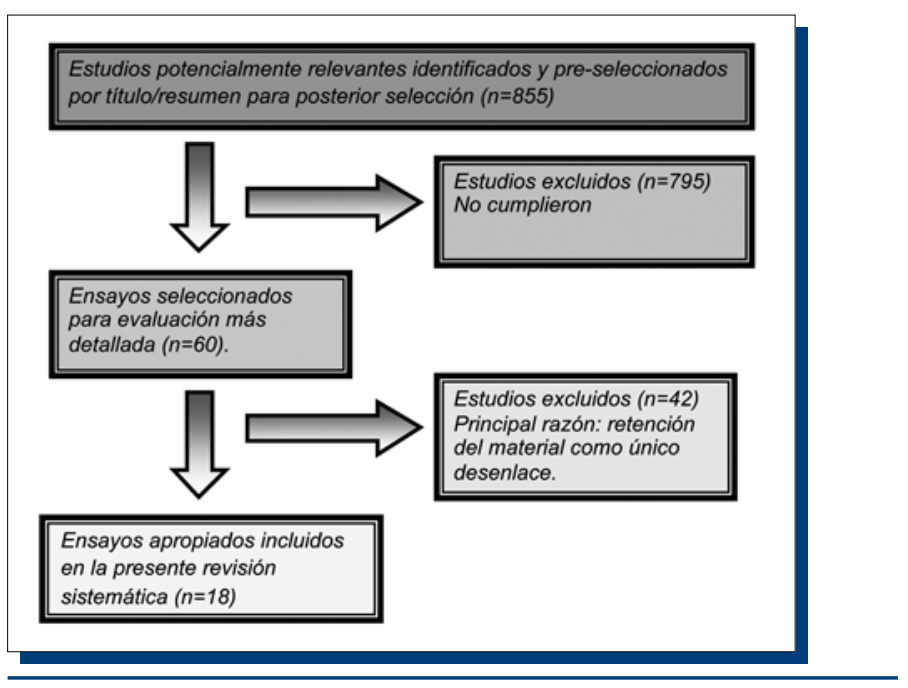

Figura 1. Estrategia de búsqueda de ensayos clínicos.

\section{DISCUSIÓN}

Luego de analizar los estudios se puede observar que todos ellos muestran una gran proporción de falencias metodológicas. Los estudios presentaron más de un $30 \%$ de incumplimiento de los puntos evaluados, llegando algunos incluso a más de un $65 \%$. Los aspectos metodológicos que faltaron con mayor frecuencia fueron: La descripción de las características basales, el enmascaramiento y la pesquisa de posibles co-intervenciones, estando la última ausente en un $94.4 \%$ de los estudios.

Con respecto al reporte de los resultados, se encontró que la gran mayoría lo realizó en forma inadecuada e incompleta, donde el porcentaje de incumplimiento de los puntos evaluados varió de un $33.3 \%$ a un $100 \%$. De los tópicos analizados en este ítem, uno de los que estuvieron ausentes en mayor proporción fue el uso de medidas de asociación o de impacto, expresándose, en la mayoría de los casos, de manera poco comprensible. También, se observó la ausencia de intervalo de confianza en muchos ensayos, así como del análisis por intención de tratamiento, análisis multinivel y el control por la presencia de cointervención. En algunos casos, la ausencia de valor $p$ de significación estadística deja en claro la no ejecución de una prueba estadística para corroborar o refutar las hipótesis, presentando los resultados en forma puramente cualitativa.

Cinco de los ensayos analizados revelaron resultados favorables a vidrio ionómero (aunque en uno de ellos no se realizó ningún método estadístico para avalar el resultado); seis fueron favorables a resina y siete estudios arrojaron resultados no significativos. En los dos estudios donde se evaluaron distintos sellantes ionoméricos, se encontró que uno de ellos reveló un resultado favorable al material convencional, mientras que el otro no entregó resultados significativos. Por lo tanto, se demuestra una inconsistencia en los hallazgos encontrados en cuanto a la comparación de estos materiales. Los principales factores que pueden haber contribuido en parte a esta situación, estarían relacionados a los aspectos metodológicos que no fueron tomados en cuenta en la etapa de diseño, pudiendo así ocasionar un deterioro irreparable de la validez interna.

En los tres estudios donde se comparó la efectividad de distintas marcas de sellantes de resina, los resultados se consideraron como no concluyentes, aun cuando el autor se inclinó hacia una marca comercial. Esto se debió, además de otras falencias metodológicas, a un insuficiente tamaño muestral, incidiendo esto negativamente en la potencia estadística, sumado a un insuficiente tiempo de seguimiento, en dos de ellos, para detectar el desenlace de interés ${ }^{(25,26)}$.

Se cree que la efectividad de los sellantes en la prevención de caries está altamente relacionada a la retención de estos materiales a la superficie del esmalte dental ${ }^{(27-30)}$. Sin embargo, la retención del material debiera ser incorporada como variable de control en el análisis de los resultados. Ninguno de los ensayos encontrados considera este procedimiento, sino que evalúan esta variable como un desenlace aislado.
La gran mayoría de los ensayos cumplieron adecuadamente con los aspectos clínicos dentro de su metodología, tales como la descripción de las intervenciones, criterios de inclusión basados en aspectos biológicos y otros, mientras que, en aspectos claves de diseño y seguimiento, se encontraron muchas carencias. Esta situación pone en evidencia una escasa aplicación de los diferentes aspectos de metodología de la investigación en este ámbito del conocimiento odontológico. Esto podría eventualmente deberse a la predominancia de investigadores eminentemente clínicos y a la carencia de equipos multidisciplinarios compuestos además por epidemiólogos y bioestadísticos.

Por otro lado, estos hallazgos ponen al descubierto la perpetuación de la aplicación indiscriminada de métodos de prevención y control de lesiones de caries, sin realizar una evaluación crítica del tema relacionado o aclarar si realmente estas medidas son realmente efectivas. Una de las explicaciones a este fenómeno estaría probablemente dada a que el profesional clínico, en general, tiende a aplicar intervenciones basándose especialmente en los resultados de la literatura publicada, no indagando mayormente en las bases que los sustentan, lo que pone a la palestra, la necesidad de una formación sólida para interpretar adecuadamente la literatura publicada, de manera que se pueda evaluar la calidad de la información publicada. Vale destacar que, además del ámbito puramente clínico, el resultado de la interpretación de los estudios puede tener fuertes implicancias en la toma de decisiones en el ámbito de la Salud Pública y, por ende, en la forma de administración y asignación de los recursos económicos y humanos, habitualmente escasos o limitados.

En el año 2004, Ahovuo-Saloranta et al.(36) publicaron una revisión sistemática acerca de la efectividad de los sellantes de resina de segunda, tercera y cuarta generación, como también de materiales ionoméricos para la prevención de lesiones de caries. Para esto se realizó una búsqueda de ensayos en el Cochrane Oral Health Group's Trial Register, Embase, Medline y otros, entre los años 1974 y 2002. Se concluyó que la efectividad de resinas versus vidrio ionómero fue contradictoria, lo que coincide con lo encontrado en la presente revisión, a pesar de que los hallazgos demostraron una tendencia más favorable a las resinas, lo que fue considerado poco convincente por los autores. El mismo autor concluye, en el año $2008^{(10)}$, que los resultados son conflictivos al compararse los distintos materiales utilizados como sellantes de fosas y fisuras, pese a que estos constituyen una estrategia efectiva de control de lesiones en grupos de alto riesgo de caries.

Beiruti y col..$^{(37)}$, en el año 2006, publicaron una revisión sistemática de los efectos preventivos de sellantes en base a resina y a ionómeros utilizando bases de datos de Medline y Cochrane review. Se incluyeron estudios hasta el año 2004. Los autores no encontraron un patrón obvio con respecto al efecto preventivo de los dos tipos de materiales, llegando a la conclusión, tal como en la presente revisión, de que no existe evidencia convincente para afirmar que las resinas son más efectivas para prevenir caries en comparación con los ionómeros y viceversa.

Simonsen ${ }^{(38)}$, en 1996, publicó una revisión crítica acerca del uso de vidrio ionómero como sellante de fosas y fisuras. Basado en los estudios analizados, el autor concluyó que la retención de los sellantes resinosos fue superior a la de vidrio ionómero, pero la diferencia en la prevención de caries permanece incierta.

En una revisión sistemática de la efectividad de los sellantes de fosas y fisuras publicada en el año 2003, Méjare y col. $^{(39)}$, analizaron estudios experimentales y cuasi-experimentales en población adolescente mayor de 14 años y concluyeron que la evidencia disponible acerca del verdadero efecto protector de estos materiales en la prevención de caries es altamente limitada, tanto para sellantes de resina como para los materiales ionoméricos.

En las revisiones sistemáticas anteriormente citadas, los autores no realizan en profundidad un análisis de aspectos de diseño y metodológicos de los ensayos incluidos, pudiendo invalidar los resultados encontrados por la falta de acuciosidad en valorar estos aspectos de gran relevancia. En el caso de la revisión realizada por Simonsen et al., por ejemplo, el análisis toma en cuenta sobretodo aspectos clínicos y de las propiedades biológicas de los materiales. Además, a diferencia de las revisiones anteriormente citadas, el presente estudio tomó en consideración la producción científica latinoamericana, mediante la búsqueda en la base de datos Lilacs, con el fin de contextualizar y analizar los hallazgos en un escenario más próximo a la realidad local.

Por otro lado, dentro de los ensayos analizados en nuestro estudio, la gran mayoría no menciona puntos importantes referentes a 
los posibles factores de confusión que podrían estar relacionados a la incidencia de caries en las poblaciones estudiadas. Las características basales de los participantes, como nivel socioeconómico, hábitos de higiene bucal, dieta, prevalencia de caries al inicio del estudio y estado de fluoración del agua de abastecimiento comunitario, no fueron mencionadas en muchos de ellos. Tampoco se menciona claramente la pesquisa de procedimientos preventivos paralelos o co-intervenciones durante el periodo de seguimiento, salvo en un solo ensayo ${ }^{(21)}$.

Un problema de suma relevancia identificado en la presente revisión tiene relación con la definición del desenlace de interés. Algunos de los ensayos no contaron con un criterio de detección bien definido para caries dental, y algunos de los que mencionaron algún criterio, no siguieron un estándar claro para dicha definición ${ }^{(11,20,21,22,30,31)}$. Este inconveniente podría resultar tanto en una mayor variabilidad de los hallazgos, así como en una incertidumbre acerca de la verdadera efectividad del material estudiado, ya que no se sabe si los autores engloban la definición de caries como lesiones incipientes en esmalte o también lesiones cavitadas en dentina.

En cuanto a la asignación aleatoria de los participantes, se encontró que la mayoría de los ensayos la menciona, pero no se deja claro el proceso utilizado para ello. Idealmente se debe expresar en forma clara el método, debido a que muchos autores consideran que es suficiente con referirla, no teniendo, en realidad, una visión correcta de lo que esto constituye ${ }^{(40)}$.

En todos los ensayos se utilizó el molar o superficie dental como unidad de análisis estadístico. Sin embargo, ninguno de ellos mencionó indicadores de dependencia entre dientes o pares de dientes en un mismo individuo, como el índice de correlación intra-clase o la estructura jerárquica de datos a través de la utilización del enfoque de análisis multinivel. El nivel dentro del cual se asignan aleatoriamente las intervenciones conlleva implicancias sobre la variabilidad de los resultados, que puede verse afectada por el azar en mayor o menor proporción, así como sobre el procedimiento de muestreo, es decir, al asignarse aleatoriamente por conglomerados, se aplica un criterio de restricción que afecta la variabilidad, aumentándola y, por ende, se debe incrementar el número de unidades de análisis ${ }^{(41)}$.
La gran proporción de fallas metodológicas importantes y la alta heterogeneidad encontrada en los estudios incluidos en la presente revisión, imposibilitan la realización de un meta-análisis. Adicionalmente, las diferencias aquí observadas también podrían estar en cierto grado influenciadas por el contexto cultural y social de los distintos estudios, así como por el enfoque teórico y metodológico del investigador, elementos importantes dentro del contexto de investigación odontológica en Salud Pública.

De esta forma, y luego de realizar esta revisión, se considera que la evidencia que avala la utilización de esta medida preventiva es metodológica y estadísticamente deficiente, mostrando de esta manera, una falencia en el desarrollo científico del área de investigación dentro de este ámbito del conocimiento odontológico. Estos hallazgos debieran estimular a los profesionales a orientar sus esfuerzos en esclarecer la verdad referente a la efectividad de los sellantes como medida preventiva antes de recomendar indiscriminadamente su utilización y/o, al menos, difundir la no existencia de evidencia consistente que demuestre el verdadero impacto de estos en la reducción de la incidencia de lesiones de caries en la población, especialmente a largo plazo.

Frente a esta realidad, se recomienda la realización de nuevos ensayos clínicos ejecutados con un adecuado diseño metodológico y de análisis estadístico, cuyos resultados sirvan como evidencia confiable para la toma de decisiones que guíen las políticas de Salud Pública Bucal, con el fin de cubrir la necesidad de conocer y aplicar la mejor intervención posible a nuestra población infantil.

\section{CONFLICTOS DE INTERÉS}

Los autores declaran no tener conflictos de interés.

\section{REFERENCIAS BIBLIOGRÁFICAS}

1. World Health Organization. Oral Health. Consultado el 12 de Marzo 2012. Disponible en: http://www.who.int/mediacentre/factsheets/fs318/es/index.html 2. Arrow P. Cost minimilization analysis of two occlusal caries preventive programmes. Community Dent Health, 2000; 17: 85-91.

3. Sheiham A. Dietary effects on dental diseases. Public Health Nutrition, 2001; 4 : 569-591.

4. Urbina T, Caro JP, Vicent M. Caries dentaria y fluorosis en niños de 6 a 8 años y 12 años. Ministerio de Salud, Chile 1996-1999. División Protección de Salud. Departamento Odontológico. Consultado el 04 de Abril 2012. Disponible en: http:// www.redsalud.gov.cl/archivos/salud_bucal/perfilepidemiologico.pdf.

5. Soto L, Tapia R y col. Diagnóstico Nacional de Salud Bucal de los niños de 6 años. Chile, 2007. Ministerio de Salud de Chile. División Protección de Salud. Departamento Odontológico. Consultado el 04 de Abril 2012. Disponible en: http:// www.redsalud.gov.cl/archivos/salud bucal/perfilepidemiologico.pdf

6. Baratieri LN, Andrada MAC, Júnior SM y cols. Operatoria dental. Editora Quintessence, 1993; 509.

7. Carvalho JC, Thylstrup A, Ekstrand KR. Dental plaque and caries on occlusal surfaces of first permanent molars in relation to stage of eruption. J Dent Res, 1989; 68: 773-779.

8. Barrancos M. Operatoria dental-integración clínica. Editorial Médica Panamericana, cuarta edición, 2006; 1603

9. Skartveit L, Tveit AB, Totdal B y cols. In vivo fluoride uptake in enamel and dentin from fluoride-containing materials. ASDC J Dent Child, 1990; 57: 97-100.

10. Ahovuo-Saloranta A, Hiiri A, Nordblad A, Worthington H, Makela M. Pit and fissure sealants for preventing dental decay in the permanent teeth of children and adolescents. Cochrane Database Syst Rev, 2008 (4): CD001830.

11. Karlzen-Reurving G, Van Dijken J. A 3-years follow-up of glass ionomer cement and resin fissure sealant. ASDC J Dent Child, 1995; 62: 108-110.

12. Forss $\mathrm{H}$, Halme $\mathrm{E}$. Retention of a glass ionomer cement and a resin-based fissure sealant and effect on carious outcome after 7 years. Community Dent Oral Epidemiol, 1998; 26: 21-25.

13. Poulsen S, Beiruti N, Sadat N. A comparison of retention and the effect on caries of fissure sealing with a glass-ionomer and a resin-based sealant. Community Dent Oral Epidemiol, 2001; 29(4): 298-301.

14. Moher D, Schulz K, Altman D. The CONSORT statement: Revised recommendations for improving the quality of reports of parallel-group randomized trials. JAMA, 2001; 285(15): 1987-1991.

15. Straus S, Richardson W, Glasziou P y cols. Medicina basada en la evidencia. Tratamiento. Editora Elsevier, tercera edición, 2006; 313.
16. Hulley S, Cummings S, Browner W y cols. Designing clinical research. Lippincott Williams \& Wilkins. Segunda edición, 2001; 336

17.Beiruti N, Frencken J, Van't Hof M, Taifour D, Van Paleinstein H. Cariespreventive effect of a one-time application of composite resin and glass ionomer sealants after 5 years. Caries Res, 2006; 40(1): 52-59.

18. Pousen S, Lauberg L, Vaeth M, Jensen U, Haubek D. A field trial of resin-based and glass-ionomer fissure sealants: Clinical and radiographic assessment of caries. Community Dent Oral Epidemiol, 2006; 34(1): 36-40.

19. Pardi V, Pereira A, Mialhe F y cols. A 5-year evaluation of two glass-ionome cements used as fissure sealants. Community Dent Oral Epidemiol, 2003; 31(5) 386-391.

20. Villela L, Fava M, Vieira M. Avaliação clínica de vinte e quatro meses do fluoroshield e do vitremer utilizados como selante de fossas e fissuras. Rev Odontologia Univ São Paulo, 1998; 12(4): 383-387.

21. Mark M, Descheper E, Dean J. Using a resin-modified glass ionomer as an occlusal sealant: A one year clinical study. Journal Am Dental Association, 1996; 127: 1508-1513.

22. Silva R, Araújo M, Rego M. Avaliação clínica de selantes de fossas e fissuras: Efeito de materiais e tempo de análise. Rev Odontologia UNESP, São Paulo, 1996; 25(2): 237-245.

23. Arrow P, Riordan P. Retention and caries preventive effects of a GIC and a resin-based fissure sealant. Community Dent Oral Epidemiol, 1995; 23(5): 282-285. 24. Songpaisan $Y$, Bratthall D, Phantumvanit $P$, Somidhivej $Y$. Effects of glass ionomer cement, resin-based pit and fissure sealant and HF applications on occlusal caries in a developing country field trial. Community Dent Oral Epidemiol, 1995; 23(1): 25-29.

25. Zuanon A, Vertuan V, Andrade M. Comparação de 5 selantes oclusais quanto à retenção e eficácia na prevenção de cáries. Rev Odontologia UNESP, São Paulo 1995; 24(1): 79-86.

26. Nóbrega P, Vertuan V. Eficácia e retenção de selantes oclusais fotopolimerizáveis Rev Odontología UNESP, São Paulo, 1994; 23(2): 253-259.

27. Forrs H, Saarni U, Seppa L. Comparison of glass-ionomer and resin-based fissure sealants: A 2-year clinical trial. Community Dent Oral Epidemiol, 1994; 22(1): $21-24$

28. Barja-Fidalgo F, Maroun S, Oliveira BH. Effectiveness of glass ionomer cement used as a pit and fissure sealant in recently erupted permanent first molars. $J$ Dent Child, 2009; 76: 34-40.

29. Kervanto-Seppala S, Lavonius E, Pietila I et al. Comparing the caries preventive effect of two fissure sealing modalities in public health care: A single application of 
glass ionomer and a routine resin based sealant program. A randomized split-mouth clinical trial. Int J Paedriatic Dent, 2008; 18: 56-61.

30. Dhar V, Chen R. Evaluation of resin based and glass ionomer based sealants placed with or without tooth preparation - a two year clinical trial. Pediatric Dentistry, 2012; 34(1): 46-50.

31. Antonson S, Antonson D, Brener $S$ et al. Twenty-four month clinical evaluation of fissure sealants on partially erupted permanent first molars. JADA, 2012; 143: 115-121.

32. Borges BCD, Campos GBP, Silveira ADS y cols. Efficacy of a pit and fissure sealant in arresting dentin non-cavitated caries: A 1-year follow-up, randomized, single-blind, controlled clinical trial. Am J Dent, 2010; 23: 311-316.

33. Muller-Bolla M, Lupi-Pégurier L, Tardieu C y cols. Retention of resin based pit and fissure sealants: A systematic review. Community Dent Oral Epidemiol, 2006 34: 321-336

34. Feigal R, Donly K. The use of pit and fisure sealants. Pediatr Dent, 2006; 28 : $143-150$

35. Locker D, Jokovic A, Kay E. Prevention. Part 8. The use of pit and fissure sealants in preventing caries in the permanent dentition of children. Br Dent J, 2003; 195: $375-378$
36. Ahovuo-Saloranta A, Hiiri A, Nordblad A, Worthington H, Makela M. Pit and fissure sealants for preventing dental decay in the permanent teeth of children and adolescents. Cochrane Database Syst Rev, 2004(3): CD001830. Review. PMID: 15266455.

37. Beiruti N, Frencken J, Van't Hof M, Van Paleinstein H. Caries-preventive effect of resin-based and glass ionomer sealants over time: A systematic review. Community Dent Oral Epidemiol, 2006; 34: 403-409.

38. Simonsen R. Glass lonomer as fisure sealant - a critical review. J Public Health Dent, 1996; 56(3): 146-149.

39. Mejàre $I$, Lingstron $P$, Peterson $L y$ cols. Caries-preventive effect of fissure sealants: A systematic review. Acta Odontol Scand, 2003; 61(6): 321-330.

40. Dawson B, Trapp R. Bioestadística médica. Ed. Manual Moderno, tercera edición, 2002; 435.

41. Donner A, Klar N. Design and analysis of cluster randomization trials in health research. Hodder Arnold Publication, 2000; 192. 